\title{
REFERENCES
}

1. I. Heller, The travelling salesman's problem I, George Washington University, Logistics Research Project Report, 1954, 88 pp.

2. E. G. Straus, Functions periodic modulo each of a sequence of integers, Duke Math. J. vol. 19 (1952) pp. 379-395.

3. F. Supnick, On the dense packing of spheres, Trans. Amer. Math. Soc. vol. 65 (1949) pp. 14-26.

4. - Optimal closed paths through certain point sets, Bull. Amer. Math. Soc. Abstract 62-1-34.

University of California, Los ANGeles

\section{CONJUGATES IN DIVISION RINGS}

\section{N. HERSTEIN}

In this note we prove the

TheOREM. If in a division ring $D$ an element $a \in D$ has only a finite number of conjugates in $D$ then it has only one conjugate, that is, $a$ is in $Z$, the center of $D$.

This theorem, of course, generalizes the famous theorem of Wedderburn which asserts that a finite division ring is a commutative field; however, since Wedderburn's theorem is used in the proof it does not yield a new proof of the result of Wedderburn. We also exhibit two corollaries to the theorem which may be of some independent interest; the second of these extends the result that a polynomial over a field having more roots than its degree in some extension field must be identically zero to a suitable analogue when the roots lie in a division ring.

PROOF OF THE THEOREM. We use the following convention throughout: if $K$ is a division ring then $K^{\prime}$ will be the group of its nonzero elements under the multiplication of $K$.

Let $a \in D$ have a finite number of conjugates in $D$. Thus if $N$ $=\{x \in D \mid x a=a x\}$ then $N$ is a subdivision ring of $D$; moreover $N^{\prime}$ is of finite index in $D^{\prime}$. Thus $N^{\prime}$ has a finite number of conjugates in $D^{\prime}$. Consequently $N$ has a finite number of conjugates in $D$, say $N=N_{1}$, $N_{2}, \cdots, N_{k}$; of course these $N_{i}$ 's are subdivision rings of $D$. Since the $N_{i}^{\prime}$ 's are all of finite index in $D^{\prime}$ and there are a finite number of them, their intersection, $T^{\prime}$, is also of finite index in $D^{\prime}$; in addition $T^{\prime}$ is normal in $D^{\prime}$. Thus $T$, the intersection of the $N_{i}$ is a subdivision

Received by the editors January 16, 1956. 
ring of $D$ invariant under all the inner automorphisms of $D$. By the Brauer-Cartan-Hua theorem [1] either $T=D$ or $T \subset Z$, the center of $D$. If $T=D$, then $N=D$ and so $a$ is in $Z$. So we consider the second possibility, namely $T \subset Z$. But since $T^{\prime}$ is of finite index in $D^{\prime}$, the fact that $T \subset Z$ implies that $Z^{\prime}$ is of finite index in $D^{\prime}$.

If $Z$ is a finite field then since $Z^{\prime}$ is of finite index in $D^{\prime}$ it follows that $D$ is a finite division ring, and so is commutative by Wedderburn's theorem.

So we suppose that $Z$ has an infinite number of elements. Consider the elements $a_{0}=a, a_{1}=a+z_{1}, \cdots, a_{n}=a+z_{n}, \cdots$ where the $z_{i}$ are an infinite number of distinct elements of $Z$. Since the index of $Z^{\prime}$ in $D^{\prime}$ is finite, for some $z_{i} \neq z_{j}, a_{i}$ and $a_{j}$ must be in the same coset of $Z^{\prime}$; that is $a+z_{i}=z\left(a+z_{j}\right)$ where $z \in Z$. Since $z_{i} \neq z_{j}, z$ can not be equal to 1 ; but then $(1-z) a=z z_{j}-z_{i}$ and so is in $Z$. Since $1-z$ is in $Z$ and is not 0 it has an inverse in $Z$, from which we deduce that $a \in Z$, proving the theorem.

COROLLARY 1. Let $D$ be a division ring with center $Z$ and suppose that $p(x)=\alpha_{0} x^{n}+\alpha_{1} x^{n-1}+\cdots+\alpha_{n}$ where the $\alpha_{i}$ are in $Z$, has one root in $D$ which is outside of $Z$. Then $p(x)$ has an infinite number of roots in $D$.

Proof. Let $a \in D, a \notin Z$ be a root of $p(x)$; then all the conjugates of $a$ in $D$ are also roots; since $a \notin Z$ it has an infinite number of conjugates, proving the theorem.

Corollary 2. Let $D$ be a division ring with center $Z$ and suppose that $p(x)$ is a polynomial of degree $n$ with coefficients in $Z$. If $p(x)$ has $n+1$ roots in $D$ then it has an infinite number of roots in $D$.

Proof. $p(x)$ has at most $n$ roots in $Z$ since $Z$ is a field, thus since it has $n+1$ roots in $D$, one of these roots must fall outside $Z$, so the corollary reduces to Corollary 1 .

\section{REFERENCES}

1. Richard Brauer, On a theorem of $H$. Cartan, Bull. Amer. Math. Soc. vol. 55 (1949) pp. 619-620.

University of Pennsylvania 